\title{
Personalisierte Medizin: Motor der Tumortherapie oder uneinlösbares Versprechen?
}

\author{
Verbessert die personalisierte Medizin die Versor- \\ gung von Patienten mit Tumorerkrankungen wirk- \\ lich? Oder werden die Erwartungen, die sich für \\ Patienten mit maßgeschneiderten Therapien verbin- \\ den, eher nicht erfüllt? Zu diesen Fragen fand im \\ Rahmen der Jahrestagung eine Pro-Contra-Debatte \\ unter der Moderation von Prof. Matthias Schwab \\ vom Dr. Margarete Fischer-Bosch-Institut für Klini- \\ sche Pharmakologie, Stuttgart, statt.
}

Hinter dem Schlagwort der personalisierten Medizin steht die Idee, das Wissen um die molekularen Prozesse des Lebens und die individuellen Patientencharakteristika insbesondere in der Onkologie für eine maßgeschneiderte Prävention, Diagnose und Therapie nutzbar zu machen.

\section{Schwache Datenlage - zu hohe Kosten}

PD Dr. Dr. Martin Müller, Universitätsklinikum Tübingen, vertrat in der Pro-Contra-Debatte die Ansicht, dass die derzeitige Datenlage das Ausmaß, in dem für den Einsatz der Strategie zur Tumorbehandlung geworben werde, keinesfalls rechtfertige: „Die personalisierte Medizin ist ein Hype“, so der Hämato-Onkologe: „Zumindest dann, wenn unter dem Schlagwort der Einsatz zielgerichteter Therapien gegen im Tumorgenom detektierte Aberrationen unabhängig vom histologischen Ursprung des Tumors verstanden wird. Hierzu existieren nur

\footnotetext{
Neues Adjuvans für Krebsimpfstoffe

So erfolgreich die bisherige Entwicklung der Checkpoint-Inhibitoren auch ist, nur ein Teil der Krebspatienten wird vermutlich von diesem immunonkologischen Ansatz profitieren, wie Prof. Hans-Georg Rammensee, Universitätsklinikum Tübingen, berichtete. So ruht weiterhin die Hoffnung auf der Entwicklung von Krebsimpfstoffen. Die Schwierigkeit besteht allerdings meist darin, dass gerade bei therapeutischen Impfstoffen, die aus mehreren Peptiden bestehen, die Immunantwort nur schwach ausgeprägt ist, wenn sie nicht gleichzeitig mit einem Adjuvans verabreicht werden. Versuche etwa mit Montaniden wie ISA51 waren bisher nicht zufriedenstellend. Die Arbeitsgruppe um Rammensee hat deshalb ein neues Adjuvans entwickelt, das Lipopeptid XS15. Der Vorteil: Die für die Vakzinierung verwendeten Peptide verbinden sich nicht kovalent mit den Lipopeptiden des Adjuvans, sondern werden von ihnen nur umschlossen.

Getestet wurde das Adjuvans bei Gesunden und unter anderem bei Patienten mit einem myelodysplastischen Syndrom. Wie Rammensee berichtete, induzieren Peptidvakzine, die mit dem XS15 verabreicht werden, eine starke CD8- und CD4-T-Helferzellantwort nach nur einer einzigen Injektion. Die Peptide seien noch mindestens sieben Wochen nach der Vakzinierung nachweisbar, und die Immunantwort halte mindestens ein Jahr an. Studien mit dem neuen Adjuvans seien geplant. Die Zukunft der Tumorvakzinentwicklung liege darin, die geeigneten patientenspezifischen Peptide zu finden, mit einem starken Adjuvans zu verabreichen und Patienten mit fortgeschrittenen Tumoren danach am besten noch mit Checkpoint-Inhibitoren zu behandeln.

Peter Leine
}

wenige klinische Daten“, kritisierte Müller. Allein aus der molekularen Identifizierung behandelbarer Mutationen den OffLabel-Gebrauch der entsprechenden zielgerichteten Substanzen in der Routineversorgung abzuleiten, sei auf Basis der derzeitigen Evidenz aus Studien nicht zu rechtfertigen. Dazu kämen die immensen Kosten für die personalisierte Medizin und die Gefahr, dass etablierte Standardtherapien zu leicht übergangen werden.

Ergänzend gab Müller zu bedenken, dass ein großer Teil der zielgerichteten Wirkstoffe lediglich eine partielle Inhibition des entsprechenden Signalwegs vermittle, eine Kombination verschiedener Wirkstoffe aufgrund von Toxizitätsproblemen aber ausgeschlossen sei. Müller betonte jedoch auch, dass die Wirksamkeit zielgerichteter Therapien gegen bestimmte molekulare Strukturen bei definierten Tumorentitäten durch klinische Studien gut belegt und ihr Einsatz in diesem Kontext selbstverständlich sinnvoll sei: „Generell sollte das Potenzial der personalisierten Medizin für präzise definierte Entitäten in diesem Sinne weiter in Studien evaluiert werden“, forderte der Hämato-Onkologe.

\section{Eine klare Erfolgsgeschichte}

Prof. Stefan Fröhling, Nationales Centrum für Tumorerkrankungen (NCT) Heidelberg, vertrat dagegen die Ansicht, dass eine tiefe und breite molekulare Charakterisierung individueller Tumorerkrankungen großes Potenzial besitze, um letztendlich die Behandlung eines sehr großen Spektrums an Krebskrankheiten zu verbessern. „Die personalisierte Medizin - oder anders gesagt die Biomarker-stratifizierte Onkologie, Präzisionsmedizin oder Präzisionsonkologie - ist keine neue Strategie. Genetische Analysen bestimmen das klinische Handeln in der Onkologie schon lange, wie etwa bei der chronischen myeloischen Leukämie mit dem Philadelphia-Chromosom. Mit dessen Entdeckung wurde die Behandlung der Erkrankung revolutioniert", sagte der Biochemiker und Mediziner. Die personalisierte Medizin sei eine Erfolgsgeschichte.

Eine tiefere und breitere genetische Analytik sei sinnvoll, weil mittlerweile zwar die meisten häufigen tumortreibenden Mutationen bekannt sind, nicht jedoch die rekurrenten Treibermutationen, die bei weniger als $5 \%$ der Patienten in einer klassischen Tumorentität vorkommen. „Ein solcher Ansatz, mit dem seltene Treibermutationen erfasst und komplexe Biomarker abgebildet werden, eröffnet zusätzliche therapeutische Chancen, gerade auch für jüngere Patienten mit fortgeschrittenen, austherapierten seltenen Tumorerkrankungen, für die kein Behandlungsstandard existiert, wie zum Beispiel Knochen- und Weichgewebesarkome“, so Fröhling. Er sei der Ansicht, betonte Fröhling abschließend, dass in nicht allzu ferner Zukunft auch chirurgische Eingriffe von genetischer Stratifizierung profitieren könnten.

Dr. Silke Wedekind

Jahrestagung der Deutschen, Österreichischen und Schweizerischen Gesellschaften für Hämatologie und Medizinische Onkologie, 29.9. bis 3.10.2017 in Stuttgart 